\title{
Electrical and spectroscopic diagnostic of an atmospheric double arc argon plasma jet
}

\author{
X Tu${ }^{1,3}$, B G Chéron ${ }^{2}$, J H Yan ${ }^{1}$ and K F Cen ${ }^{1}$ \\ ${ }^{1}$ Institute for Thermal Power Engineering, State Key Laboratory of Clean Energy Utilization, \\ Zhejiang University, Hangzhou 310027, People's Republic of China \\ ${ }^{2}$ UMR 6614 CNRS CORIA, Saint Etienne du Rouvray 76801, France \\ E-mail: plasmas@zju.edu.cn
}

Received 21 June 2007, in final form 23 August 2007

Published 3 October 2007

Online at stacks.iop.org/PSST/16/803

\begin{abstract}
An atmospheric argon plasma jet generated by an original dc double anode plasma torch has been investigated through its electrical and spectroscopic diagnostics. The arc instabilities and dynamic behavior of the argon plasma are analyzed using classical tools such as the statistical method, fast Fourier transform (FFT) and correlation function. The takeover mode is identified as the fluctuation characteristic of the double arc argon plasma jet in our experiment. The FFT and correlation analysis of electrical signals exhibit the only characteristic frequency of $150 \mathrm{~Hz}$, which originates from the torch power and is independent of any change in the operating parameters. No high frequency fluctuations $(1-15 \mathrm{kHz})$ are observed. This indicates that the nature of fluctuations in an argon plasma jet is induced mainly by the undulation of the tri-phase rectified power supply. It is found that each arc root attachment is diffused rather than located at a fixed position on the anode wall. Moreover, the emission spectroscopic technique is performed to determine the electron temperature and number density of the plasma jet inside and outside the arc chamber. Along the torch axis, the measured electron temperature and number density of the double arc argon plasma drop from $12300 \mathrm{~K}$ and $7.6 \times 10^{22} \mathrm{~m}^{-3}$ at the divergent part of the first anode nozzle, to $10500 \mathrm{~K}$ and $3.1 \times 10^{22} \mathrm{~m}^{-3}$ at the torch exit. In addition, the validity criteria of the local thermodynamic equilibrium (LTE) state in the plasma arc are examined. The results show that the measured electron densities are in good agreement with those calculated from the LTE model, which indicates that the double arc argon plasma at atmospheric pressure is close to the LTE state under our experimental conditions.
\end{abstract}

(Some figures in this article are in colour only in the electronic version)

\section{Introduction}

In the 1960s, the fundamental research devoted to thermal plasmas within the framework of the NASA aerospace program greatly motivated the development of industrial applications of thermal plasma technology in the 1980s and 1990s. Nowadays, thermal plasmas are extensively used in fields $[1,2]$ such as welding, cutting, spraying, chemical vapor

3 Author to whom any correspondence should be addressed. deposition (CVD), metallurgy, synthesis of fine powders and hazardous waste destruction due to their high temperature $(3000-15000 \mathrm{~K})$ and high energy density (up to $10^{8} \mathrm{~W} \mathrm{~m}^{-3}$ ). The most widely used sources in these processes are the plasma torches, operated in either transferred or non-transferred arc mode. Nevertheless, electrode erosion, short lifetime and unreliable performance of the plasma devices, mainly ascribed to poor understanding of the basic plasma phenomena involved, have been the major obstacles to further development in thermal plasma technology and have limited its more 
potential industrial applications. Therefore a detailed investigation to explore the important properties of the plasma source, such as the arc dynamic behavior, temperatures, specie densities and the equilibrium state, is necessary and indispensable for the optimization and improvement of the plasma devices. These properties, associated with the underlying physical phenomena of the plasma sources, will play a significant role in determining the effects of the plasma processing. For example, in the plasma vitrification process of municipal solid waste incinerator (MSWI) fly ashes, the arc unsteadiness and jet fluctuations may result in incomplete or non-uniform melting of the injected residues and, consequently, affect the physico-chemical characteristics of the final products.

In recent years, much effort has been devoted to the diagnostic of the plasma jet properties. The time-series and frequency analysis of electrical parameters (arc voltage, current intensity and input torch power), light emission and acoustic signals are the most common ways to understand the basic nature of the plasma jet fluctuations. Coudert et al [3] characterized the arc dynamic behavior of a de plasma torch by associating the arc voltage temporal evolution with arc column stretching and upstream or downstream breakdowns. In this case, the working gas is axially injected into the arc chamber along the cathode tip. Singh et al [4] investigated the influence of the controllable parameters (gas flow rate and arc current) on the arc instabilities through the fluctuations of the arc voltage and luminous intensity in a dc argon plasma jet. Dorier et al [5] focused on the arc root behavior of a dc plasma torch by simultaneous measurements of the timeseries of the arc voltage and of images from the nozzle interior. Duan and Heberlein [6], Benocci et al [7] and Zhao et al [8] also studied the arc fluctuations with different plasma spray torch configurations. Different arc fluctuation modes have been observed in relation to the thickness of the cold boundary layer between the arc column and the anode wall: 'restrike mode' with a sawtooth shape profile and high voltage fluctuation, 'takeover mode' with a more or less sinusoidal voltage waveform and relative small voltage fluctuation, 'steady mode' with very low mean voltage and weak fluctuation due to the balance between the drag force of the plasma gas and the electromagnetic forces acting on the arc attachment.

Another powerful tool extensively used for plasma investigations is optical emission spectroscopy (OES), which is non-intrusive and gives rich information about the plasma constituents and their respective energy states. By analyzing and interpreting the measured plasma spectra, we can obtain important parameters of the plasma source, such as plasma composition, temperatures, specie densities, as well as the ionization state. In the past few years, numerous works have been carried out on the study of the plasma jet properties by means of the emission spectroscopic technique [9-15].

Despite considerable research and developments in this area, a more complete understanding of the plasma jet properties is still necessary, particularly in the investigations of a newly designed plasma torch with special configuration, to improve and optimize its performance. Previous studies in our laboratory have been focused on the characterization of a supersonic low-pressure nitrogen plasma jet. These

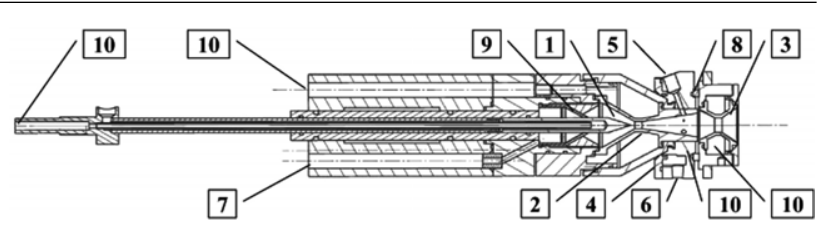

Figure 1. Configuration of the dc double anode plasma torch: (1) cathode, (2) first anode, (3) second anode, (4) torch body, (5) particle injection entrance, (6) optical fiber entrance, (7) gas inlet, (8) ceramic ring, (9) gas diffuser, (10) water cooling.

experimental works were intended to validate free jet and boundary layer kinetic schemes involved in the numerical simulation of space shuttle re-entries [16-19].

In this paper, an original dc double anode plasma torch which produces a long lifetime and highly stable atmospheric argon plasma jet has been devised for the purpose of hazardous waste treatment. The arc dynamic behavior and jet fluctuations are investigated by means of classical tools, such as the statistical method, fast Fourier transform (FFT) and correlation analysis. The emission spectroscopic technique is employed to study the key parameters of the plasma source by recording and analyzing the argon plasma spectra inside and outside the arc chamber. The excitation temperatures of the plasma arc are determined by using the Boltzmann plot method, while the electron number densities are derived from the Stark broadening of Ar I lines. In addition, the validity criteria of the local thermodynamic equilibrium (LTE) state existing in the plasma arc is discussed.

\section{Experimental setup}

\subsection{The plasma source}

The configuration of the dc double anode plasma torch used in our experiment is shown in figure 1. Compared with the conventional plasma torch, this one has a special design with two nozzle-shaped copper anodes set at different axial $(z)$ distances from the cathode tip. An alumina ceramic ring is used to ensure electrical insulation between both anodes. Its internal diameter is chosen large enough to avoid cracking on account of the high temperature arc passing through this region during the operation. The torch is supplied through two identical tri-phase rectified power sources, with different unloaded voltages of $140 \mathrm{~V}$ and $210 \mathrm{~V}$, respectively. The working gas (argon) is injected axially into the arc chamber through a gas diffuser. The gas flow rate controlled by a numerical mass flow meter (Bronkhorst High-Tech E-7000) is adjusted from 15 to $30 \mathrm{~L} \mathrm{~min}^{-1}$. The plasma is created as follows: the first arc is initiated between the tip of a coneshaped tungsten cathode $(2 \% \mathrm{Th})$ and the throat $(5 \mathrm{~mm}$ in diameter) of the first anode. Then the plasma is reheated by the second arc which is ignited between the cathode tip and the throat $(10 \mathrm{~mm}$ in diameter) of the second anode. Thus, the plasma is operated in the double arc mode and sustained between one cathode spot and two independent anode arc root attachments (figure 2). The aerodynamic stability and luminous intensity of the plasma arc as well as the jet length are greatly enhanced by the latter ignition. The same phenomenon has also been observed when the plasma is released under low pressure [19]. The electrical operating conditions are typically 


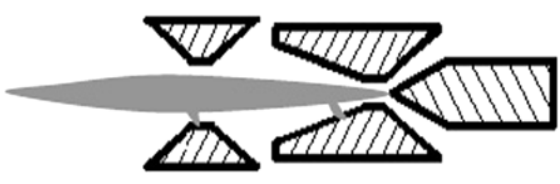

Figure 2. Plasma jet operated in double arc mode with two independent anode arc root attachments.

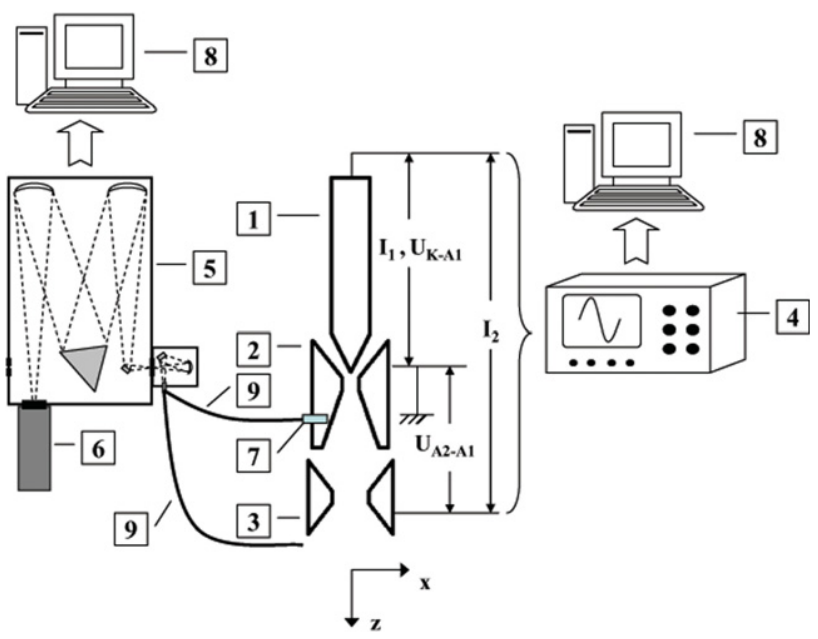

Figure 3. Schematic diagram of the diagnostic setup: (1) cathode, (2) first anode, (3) second anode, (4) oscilloscope, (5) spectrometer, (6) CCD Camera, (7) optical fiber entrance, (8) computer, (9) optical fibers.

20-30 V/80-200 A for the first arc and 50-60 V/80-200 A for the second arc. Each electrode and the torch body are water cooled independently.

\subsection{The measurement system}

Figure 3 shows a schematic diagram of the measurement setup. In order to compare the electronic parameters along the torch axis inside and outside the arc chamber at the same time, two VUV silica optical fibers with diameter $600 \mu \mathrm{m}$ are used to record the emission spectra of the plasma jet. One is located at $30 \mathrm{~mm}$ downstream from the first anode throat (divergent part of the first anode) inside the arc chamber, while the other is disposed at $10 \mathrm{~mm}$ downstream from the torch exit. Both fibers are connected to an Acton 758i spectrometer set for a $0.03 \mathrm{~nm}$ resolution within a wide spectral range from 190 to $1000 \mathrm{~nm}$.

The arc voltage and current intensity are measured simultaneously by using a four-channel digital oscilloscope (9314AL Lecroy, $400 \mathrm{MHz}, 8$ bytes) connected to the computer via GPIB. The maximum sampling rate is $100 \mathrm{MSa} \mathrm{s}^{-1}$. The voltage drop $U_{K-A 1}$ (between the cathode and the first anode) and $U_{A 2-A 1}$ (between the second and the first anode) are recorded. The voltages of both $\operatorname{arcs} U_{1}$ and $U_{2}$ can be obtained from the following expressions:

$U_{1}=-U_{K-A 1} \quad$ and $\quad U_{2}=U_{A 2-A 1}-U_{K-A 1}$

The current intensities $I_{1}$ and $I_{2}$ are recorded by using amperemetric clamps $\left(100 \mathrm{kHz}, 10 \mathrm{mV} \mathrm{A}^{-1}\right)$ set around the electrical circuits. Four thermocouples are used to measure the water temperatures at the inlet and the outlet of the cooling circuits.

\section{Results and discussion}

\subsection{Specific enthalpy and thermal efficiency}

The specific enthalpy of the plasma at the torch exit can be deduced from the temperature difference $\Delta T_{w j}$ between the inlet and the outlet of the cooling circuit, expressed as:

$$
h_{\mathrm{p}}=\frac{\sum_{i=1}^{2} U_{i} I_{i}-\sum_{j=1}^{4} q_{w j} C_{w} \Delta T_{w j}}{q_{\mathrm{g}}}+h_{0},
$$

where $h_{0}$ is the specific enthalpy of the working gas before entering the plasma torch, $U_{i}$ and $I_{i}$ represent the arc voltage and the current intensity (first and second arc), $q_{w j}, C_{w}$ and $q_{\mathrm{Ar}}$ are, respectively, the water flow rates (cathode, first anode, second anode and torch body), the water specific heat and the gas mass flow rate. In equation (2), the term of the kinetic energy contributing to the net power of the torch is completely neglected due to the low plasma velocity $\left(120-240 \mathrm{~m} \mathrm{~s}^{-1}\right)$ at the torch exit. Under our operating conditions, the specific enthalpy of the double arc argon plasma is $(5.0 \pm 1.0) \mathrm{MJ} \mathrm{kg}^{-1}$.

Another important parameter of a plasma torch is its thermal efficiency $\eta$, defined as

$$
\eta=\frac{\sum_{i=1}^{2} U_{i} I_{i}-\sum_{j=1}^{4} q_{w j} C_{w} \Delta T_{w j}}{\sum_{i=1}^{2} U_{i} I_{i}} .
$$

In our experiment, the thermal efficiency of this torch fed with pure argon at the double arc mode is $(35 \pm 3) \%$. This value is comparable to or greater than those of dc argon plasma jets in similar power conditions [20,21]. At a constant gas flow rate, this efficiency is appreciably enhanced by increasing torch power. In most plasma torches, the heat transfer through the anode wall occupies the greatest part of the total heat losses. In our case, at an argon flow rate of $20 \mathrm{~L} \mathrm{~min}^{-1}$, the heat losses on the first and the second anode contribute to $47 \%$ and $36 \%$, respectively, of the total power released by the cooling circuit, in contrast to the cathode power loss of around $4 \%$.

\subsection{Current-voltage characteristics}

One of the most important electrical parameters of the plasma arc is its current-voltage characteristics, which depend on the plasma torch configuration and the input power source. The relation between the arc voltage and the current intensity is linked to the mechanisms through which the arc column gains and loses energy. The current-voltage characteristics of a free burning arc generally exhibit a falling tendency, whereas an increasing trend may appear when the arc is crossed by high current intensity or confined by the anode wall.

In our plasma torch, the latter tendency is verified for both arcs owing to the perfect confinement of the arcs by the anode nozzle channels, as typically shown in figure 4 . Some physical considerations are sufficient to explain this phenomenon. Owing to the weak variation of the arc length, the column voltage drop can be regarded as proportional to the ratio $j / \sigma$ of the current density to the plasma electrical conductivity:

$$
U=E L=\frac{j}{\sigma} L .
$$




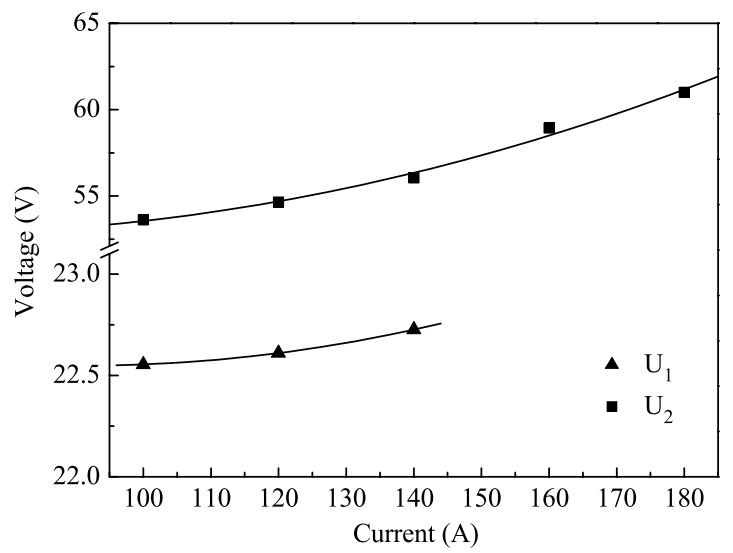

Figure 4. Current-voltage characteristics of a double arc argon plasma at a gas flow rate of $30 \mathrm{~L} \mathrm{~min}^{-1}$.

The current density increases linearly with the arc current, while the increasing current intensity also enhances the temperature and, consequently, the electrical conductivity through a non-linear law. At high temperature, the production of free electrons saturates and the current density increases faster than the electrical conductivity, thus resulting in an increase in the arc voltage. From an energetic point of view, this enhancement of the arc temperature leads to steep radial gradients: the generator must provide more power to sustain the arc at a fixed current to keep the energy balance. This increasing trend of current-voltage characteristics of the plasma arc has also been reported by Osaki et al [20], Delair [22], Hrabovsky et al [23] and Deron [24].

\subsection{Dynamic behavior}

3.3.1. Fluctuations of electrical signals. Figure 5 shows the typical time dependence of the arc voltages, current intensities and jet power at an argon flow rate of $20 \mathrm{~L} \mathrm{~min}^{-1}$. These electrical signals exhibit similar sinusoidal shapes which are governed by a $150 \mathrm{~Hz}$ characteristic frequency of the rectified power supply. As can be seen from figure 5(a), no sawtooth voltage trace ascribed to the high frequency restrike fluctuations can be observed. The time of the increase in the arc voltage, which corresponds to the arc root displacement downstream in the flow direction, is close to that of the voltage decrease associated with the upstream movement of the arc root in the direction opposed to the flow. It is noticeable that the relative fluctuation amplitudes are very low: $2-5 \%$ for the arc voltages, 6-10\% for the current intensities and jet power. It reveals small anode arc root displacement in the axial direction and quasi-constant arc column length. A phase shift between $I_{1}$ and $I_{2}$ can be noted due to the non-synchronous motion of both arc roots on the anode channels.

Figure 6 presents the variation of the mean arc voltage as a function of the argon flow rate at fixed current intensity. It is seen that the arc voltages slightly increase with the increase in the argon flow rates for both arcs. Increasing gas flow rate requires higher energy for the plasma gas heating, which in return requires a higher electric field. On the other hand, an increase in the gas flow rate causes a greater drag force which originates from the presence of the gas flow acting on the anode attachment and pushes the arc downstream. At the same time,
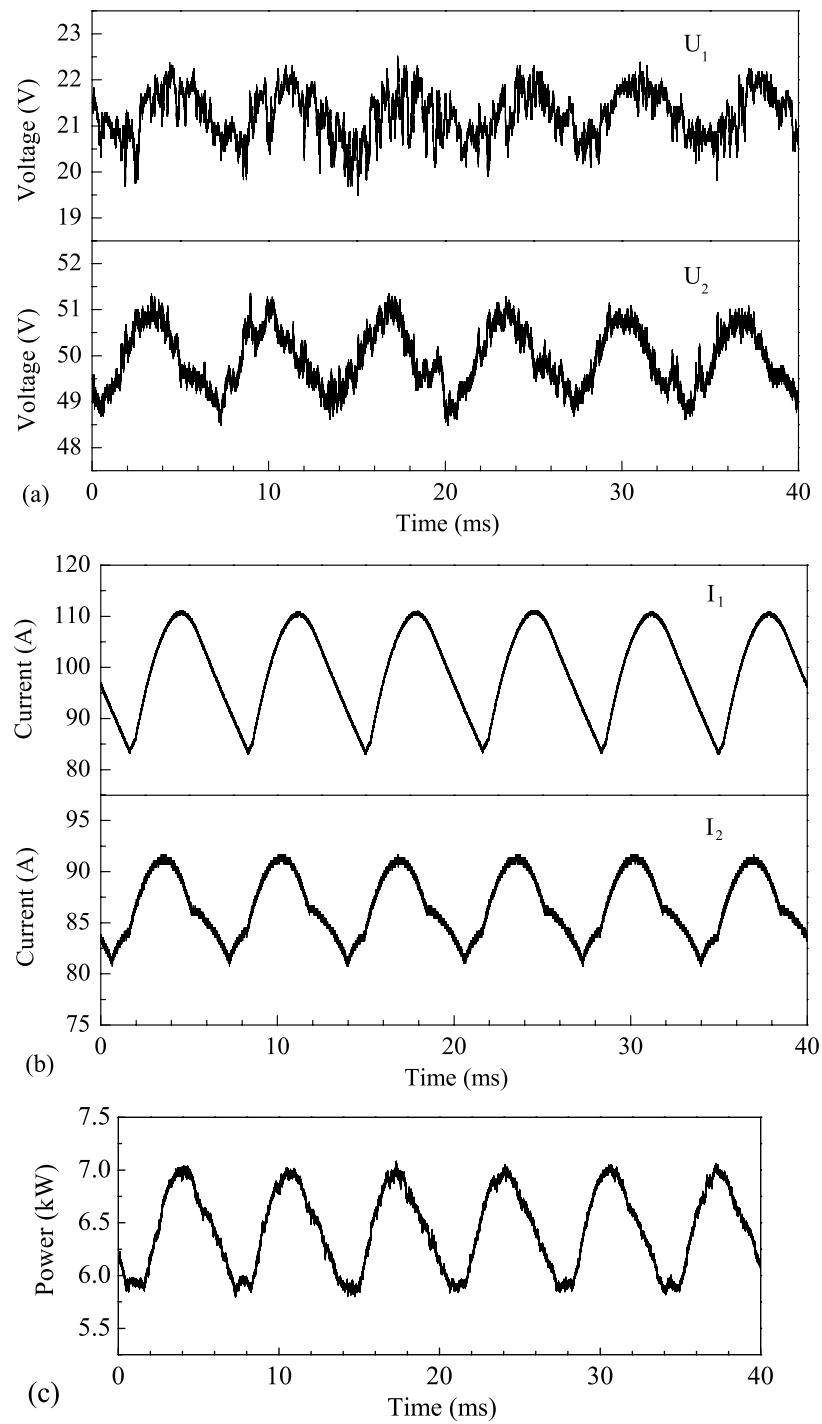

Figure 5. Time-resolved electrical signals of dc double arc argon plasma jet at a gas flow rate of $20 \mathrm{~L} \mathrm{~min}^{-1}:(a)$ arc voltages $U_{1}$ and $U_{2} ;(b)$ current intensities $I_{1}$ and $I_{2} ;(c)$ input jet power.

the arc column becomes more constricted and the cold gas boundary layer becomes thicker, which can lead to a longer arc. Both of these effects contribute to the increase in the arc voltage with rising gas flow rate.

The influence of the torch operating parameters on the arc voltage fluctuations is investigated in figure 7 . It is found that the relative voltage fluctuation amplitudes of both arcs increase with the gas flow rate, as presented in figure $7(a)$. When the gas flow rate increases, the arc turbulent intensity becomes much stronger and the anode arc root position will transfer further downstream, resulting in a rise in the voltage fluctuation and the relative fluctuation amplitude. Figure 7(b) shows the variation of the arc voltage fluctuations versus current intensities at a constant argon flow rate. The relative fluctuation amplitudes exhibit a falling tendency with the increase in current intensity. For the second arc, this effect is considerably weaker. The voltage fluctuation amplitude correlates with the arc breakdown potential, while in the arc fluctuations with the takeover mode, thermal breakdown is thus expected. This decreasing evolution in our case can be ascribed 
Electrical and spectroscopic diagnostic of an atmospheric double arc argon plasma jet
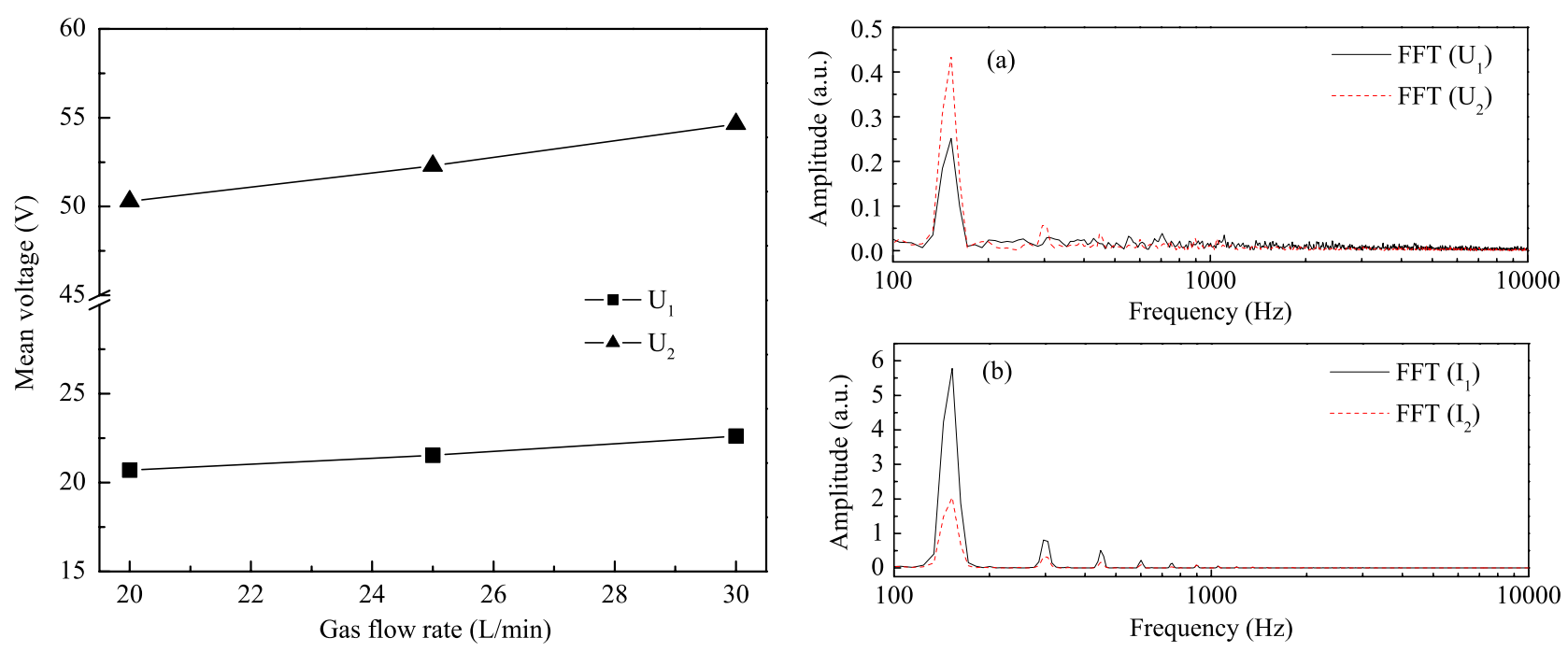

Figure 6. Variation of the mean arc voltage as a function of the $\operatorname{argon}$ flow rate $\left(I_{1}=I_{2}=100 \mathrm{~A}\right)$.
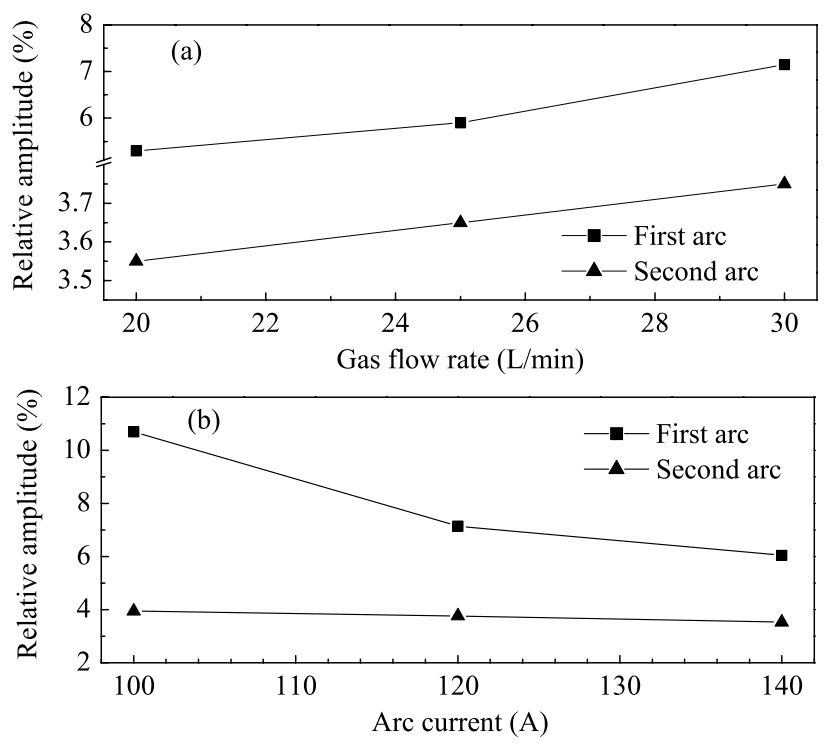

Figure 7. Influence of operating parameters on the arc voltage fluctuations: (a) relative voltage fluctuation amplitude versus argon flow rates $\left(I_{1}=I_{2}=100 \mathrm{~A}\right) ;(b)$ relative voltage fluctuation amplitude versus current intensities $\left(q_{\mathrm{Ar}}=30 \mathrm{~L} \mathrm{~min}^{-1}\right)$.

to the thickness of the arc column dimension resulting in higher thermal inertia with the increase in current intensity. It is considered that increasing the arc current results in a variation of the temperature and the density distribution and thus changes the gas dynamic properties between the arc and the anode wall. A similar behavior was reported in earlier studies by Kaminska and Dudeck [25].

From the fluctuations of these signals coupled with their statistical analysis, we can conclude that the takeover mode characterizes the fluctuating behavior of the atmospheric double arc argon plasma jet. In addition, it is worth noting that the average voltage of the second arc is much higher than that of the first arc, whereas the relative amplitude of the second arc fluctuation is smaller than that of the first one under the same operating conditions. So the corresponding

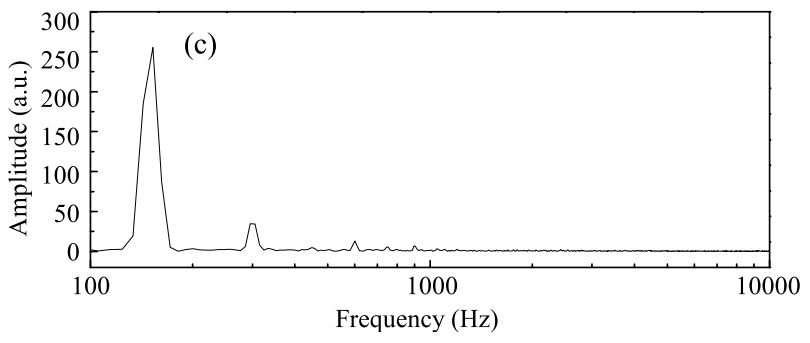

Figure 8. FFT power spectra of electrical signals shown in figure 5: $(a)$ arc voltages; $(b)$ current intensities; $(c)$ jet power.

arc root attachment is less influenced by the aerodynamics of the plasma jet than the other one. These results confirm the previous observation that the aerodynamic stabilities of the plasma jet are effectively improved with the ignition of the second arc. In our experiments, the original and special design of the plasma torch with a double anode structure provides the possibility of maintaining a much higher arc voltage and input power at low arc current.

3.3.2. FFT and correlation analysis. By using the FFT method, the amplitude spectra of the arc voltages $\left(U_{1}, U_{2}\right)$, current intensities $\left(I_{1}, I_{2}\right)$ and jet power at the argon flow rate of $20 \mathrm{~L} \mathrm{~min}^{-1}$ are obtained, as shown in figure 8 . Whatever the recorded electrical signals, the prominent peak situated at $150 \mathrm{~Hz}$ and its harmonics $(300$ and $600 \mathrm{~Hz}$ ) are clearly visible in all the spectra. These frequencies are independent of any change in the gas flow rate and the current intensity. They originate from the typical characteristic frequency of the undulation of the power supply. It means that the inherent characteristics of the torch power sources have strong effects on the arc unsteadiness and fluctuation behavior. Moreover, it should be noted that no high frequency components $(1-15 \mathrm{kHz})$ can be observed either from the amplitude spectra of electrical signals or from those of the acoustic waves of the plasma jet at the torch exit under different operating conditions. It is generally considered that such high frequency fluctuations are related either to the arc root motion on the anode wall or to the large-scale shunting of the plasma jet [3-8], with a frequency which increases with increasing gas flow rate and arc current. In some cases, the arc fluctuations induced by 

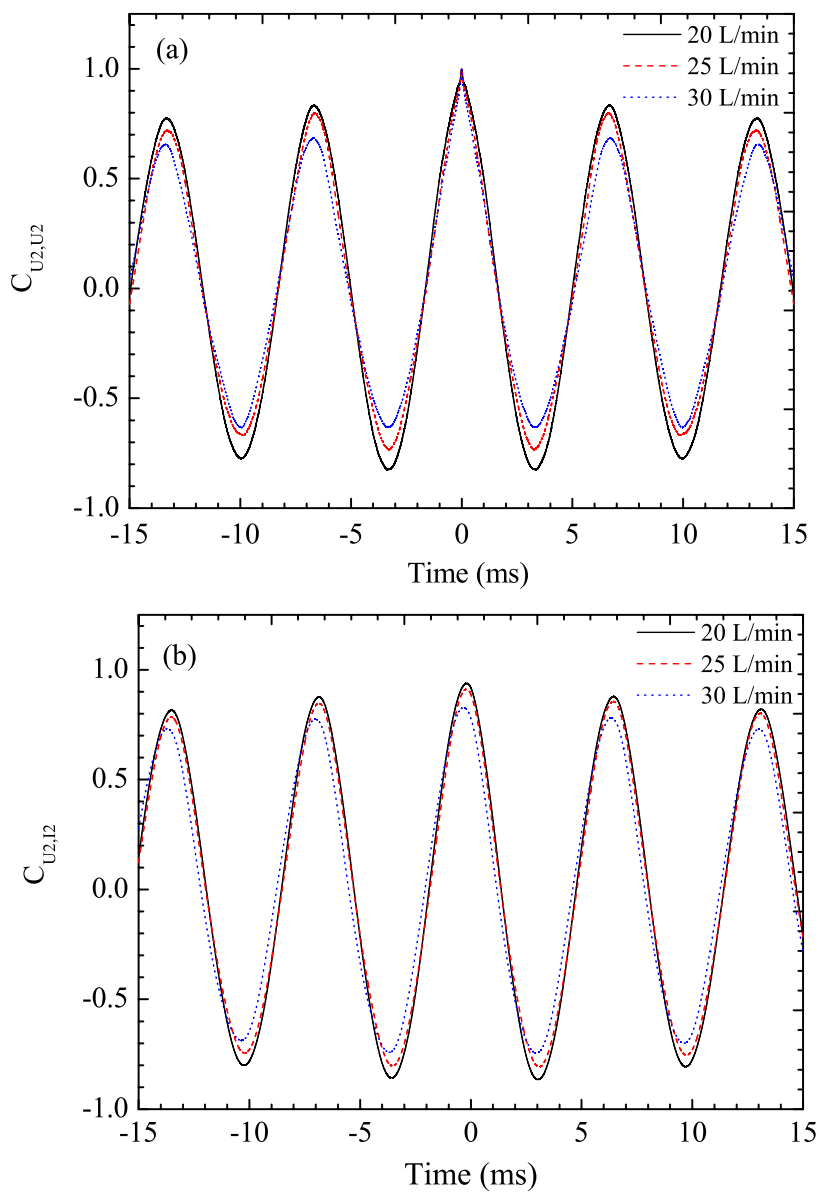

Figure 9. Time correlation calculations of electrical signals $\left(q_{\mathrm{Ar}}=20 \mathrm{~L} \mathrm{~min}^{-1}, I_{1}=I_{2}=100 \mathrm{~A}\right):(a)$ self-correlation of the arc voltage $U_{2} ;(b)$ cross correlation between the arc voltage $U_{2}$ and current intensity $I_{2}$

the dominant high frequency can be linked to severe electrode wear $[5,26]$. These promising results indicate that our torch of special design can effectively reduce the high frequency fluctuations for the case of the pure argon plasma. It is also noted that the background level of the Fourier spectra increases with the increase in the argon flow rate. This variation is also ascribed to the effect of turbulence, as previously mentioned in figure $7(a)$. When the argon flow increases from 15 to $30 \mathrm{~L} \mathrm{~min}^{-1}$, the Reynolds number at the torch exit is estimated to increase from 210 to 420 at the arc temperature of $10000 \mathrm{~K}$.

Whatever the torch operating parameters, the time correlation calculations exhibit a very regular waveform and perform alike. The influence of the gas flow rate on the selfcorrelation coefficient of the arc voltage $U_{2}$ is displayed as an example in figure $9(a)$. The successive maxima clearly correspond to high correlation of low frequency fluctuation at $150 \mathrm{~Hz}$, as expected from the Fourier spectra. It also means that the low frequency oscillations of the recorded signals are quite reproducible. No modulation of the correlation coefficients can be found at high frequency, which is in agreement with the FFT results of electrical signals. The cross correlation and selfcorrelation functions derived from the other signals also exhibit similar features. The evolution of the cross correlation between $U_{1}$ and $U_{2}$ reveals that both arcs behave as the one and only system. In figure $9(b)$, the arc voltage $U_{2}$ is found to be slightly delayed with respect to the current intensity $I_{2}$. For an argon flow rate of $20 \mathrm{~L} \mathrm{~min}^{-1}$, the delayed time is about $0.193 \mathrm{~ms}$. Such a non-synchronous phenomenon of electrical signals is expected to be attributed to the correlation between the anodic arc attachment behavior and the arc current path variation in the arc column. De Izarra et al [27] proposed that the time shift between the arc voltage and the current intensity could be reflected in the laminar-turbulent transition in the argon plasma jet. In our experiment, one notes that this value increases with the gas flow rate and decreases with the increase in current intensity. The influence of the working parameters on the time shift exhibits the same evolution as observed for those on the arc voltage fluctuations. It suggests that the time shift between the arc voltage and the current intensity can be used as an independent parameter to characterize the fluctuation behavior of the plasma jet to some extent.

Combined with the temporal series of the arc voltages, the argon plasma jet in our experiment is excited by a double arc, and neither of them is attached at single and local arc roots as is generally the case in the conventional plasma torch. The global variations of both arc voltages are a reflection of the power supply undulation, while the irregular and tiny voltage fluctuations correspond to both arc roots displacement back and forth towards the farthest (downstream) or closest (upstream) position from the cathode on the anode surface. This suggests that the movements of both anodic arc roots are erratic and weak in amplitude, which is also confirmed by a comparison between the amplitude spectra of both arcs. These arc attachments are rather evenly diffused on the anodes surface. Such a diffused arc root attachment is obviously favorable for reducing the anode erosion and prolonging the lifetime of the plasma torch. In our case, after a few hours of operation, both anodes show a very weak erosion, almost without any surface modification.

\subsection{Spectroscopic measurement}

The spectroscopic diagnostic technique is employed to measure the emission spectra of the double arc argon plasma jet between 350 and $750 \mathrm{~nm}$ inside and outside the arc chamber. The spectral scans at the first and the second stages of the plasma source are very much alike. Both spectra are clearly dominated by the numerous intensive argon atomic lines emitted in the near-UV and visible range. Some argon ionic lines are observed in the divergent part of the first anode inside the arc chamber, while these lines become very weak at the torch exit. In addition, owing to the engulfment of the ambient air, three nitrogen atomic lines (742.364, 744.229 and $746.831 \mathrm{~nm}$ ) emitted from the same energy state are observed at the torch exit, where the $\mathrm{N}_{2}^{+}$first negative system is hardly detected.

3.4.1. Excitation temperature. The excitation temperature of the plasma jet is determined using the Boltzmann plot method from the following equation:

$$
\ln \left(\frac{I_{\mathrm{nm}} \lambda_{\mathrm{nm}}}{g_{n} A_{\mathrm{nm}}}\right)=\ln (D)-\frac{E_{n}}{k_{\mathrm{B}} T_{\mathrm{e}}},
$$

where $\lambda_{\mathrm{nm}}$ is the wavelength, $A_{\mathrm{nm}}$ is the transition probability, $E_{n}$ and $g_{n}$ are the excitation energy and the statistical weight 
of the upper state respectively, $k_{\mathrm{B}}$ is the Boltzmann constant and $D$ is a constant for all the considered lines. By plotting $\ln \left(I_{\mathrm{nm}} \lambda_{\mathrm{nm}} / g_{n} A_{\mathrm{nm}}\right)$ versus $E_{n}$, we derive the excitation temperature from the slope of the Boltzmann-fitted straight line. This method does not require a standard source for the calibration, but a maximum energy range of the emitting level should be covered to get a good accuracy.

In the divergent part of the first anode, seven $\mathrm{Ar}$ II lines $(457.93,458.99,460.95,473.58,484.79,487.99$ and $500.92 \mathrm{~nm}$ ) are chosen to draw the Boltzmann diagram and derive the excitation temperature, while at $10 \mathrm{~mm}$ downstream from the torch exit, we use several Ar I lines (667.73, 675.28, $687.13,693.77,703.03,714.70$ and $727.30 \mathrm{~nm}$ ) for this study. These lines are well isolated and not self-absorbed. The spectroscopic parameters of the selected lines are taken from the atomic database of NIST [28]. The energies of the upper states for these lines spread over a wild range, which provides a good sensitivity and a least uncertainty for the measurement. Due to the involved states being populated mainly through the electron impacts, it is legitimate to equate the excitation temperature to the electronic one.

The radial evolution of the electron temperatures of a plasma arc is generally obtained using Abel inversion. However, in our study, the presence of the optical fiber-based spectroscopic diagnostic setup made it impossible to apply this method. Therefore, the plasma emission collected and transferred by each optical fiber results from the integration of the spectral intensity along the line of sight. The calculated temperature in this case is well represented by the hottest zone of the plasma flow.

Figures $10(a)$ and $(b)$ illustrate the typical Boltzmann plots obtained with the selected argon lines. As shown in figure 10, the points in the Boltzmann plots are generally well aligned and the linear fitting corresponds to a high correlation coefficient of over 0.98 , which suggests that the excitation equilibrium state of the plasma arc is well verified.

The variations show that the excitation temperature of the double arc argon plasma jet drops from $12300 \mathrm{~K}$ in the divergent part of the first anode to $10500 \mathrm{~K}$ at the torch exit. The inaccuracy of this measurement is estimated to be around 5\%. These temperatures are expected to be close to the electronic ones. The weak axial gradient of the electron temperatures indicates that the lighting of the second arc prevents the strong decay of the plasma excitation between these lines of sight.

3.4.2. Electron density. The electron density is a key parameter for determining the ionization equilibrium state of the plasma and for calculating the energy transfer in the plasma arc. In our experiment, this value is deduced from the Stark broadening of the argon atomic lines. Two well isolated Ar I lines ( 415.86 and $430.01 \mathrm{~nm}$ ) with accurately known values of the broadening parameter are used in this study.

The spectral line broadening is a complicated function of the environment of the radiating atoms or ions. The measured spectral line shape is the result of the combined effects of several broadening mechanisms. In our experiment, the total width of the spectral line is a convolution of the Lorentzian profile (Stark broadening) and the Gaussian one (Doppler broadening and instrumental broadening). For the
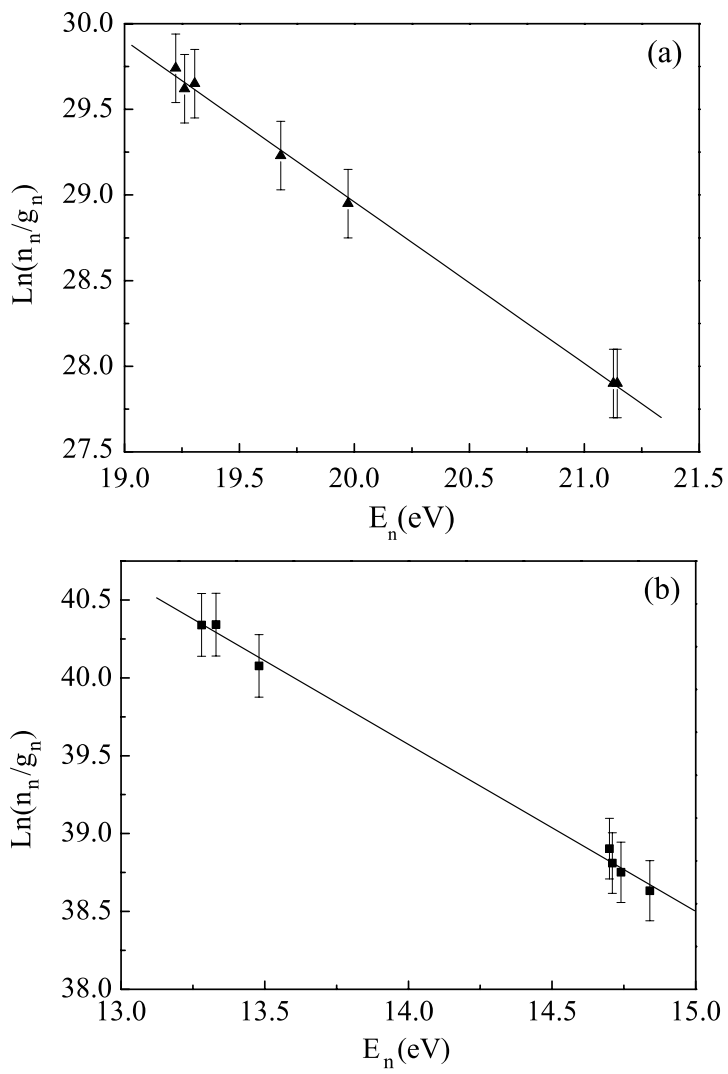

Figure 10. Typical Boltzmann plots for $(a)$ the selected Ar II emission lines (inside the arc chamber) and $(b)$ the selected Ar I emission lines (at $10 \mathrm{~mm}$ downstream from the torch exit).

temperature range (about $10000 \mathrm{~K}$ ) used in our case, the Doppler broadening is very weak and its resulting influence on the final Stark width can be neglected. Thus, the Gaussian profile only accounts for the broadening due to the apparatus function, which is determined by using a low-pressure $\mathrm{Hg}-\mathrm{Cd}$ lamp $\left(\Delta \lambda_{\text {app }}=0.03 \mathrm{~nm}\right)$. Thus, the Stark broadening (fullwidth at half-maximum, FWHM) $\Delta \lambda_{\mathrm{S}}$ of the recorded spectral line is obtained by fitting a Voigt function.

The Stark broadening $\Delta \lambda_{\mathrm{S}}$ of a well isolated nonhydrogenic line is related to the electron density $n_{\mathrm{e}}\left(\mathrm{m}^{-3}\right)$ by the semi-empirical formula proposed by Griem [29]:

$$
\begin{aligned}
\Delta \lambda_{\mathrm{S}}(\mathrm{nm}) & =2 \times 10^{-22} w n_{\mathrm{e}} \\
\times & {\left[1+1.75 \times\left(\frac{n_{\mathrm{e}}}{10^{22}}\right)^{\frac{1}{4}} \alpha(1-0.75 R)\right], }
\end{aligned}
$$

where

$$
R=8.99 \times 10^{-2} n_{\mathrm{e}}^{\frac{1}{6}} T_{\mathrm{e}}^{-\frac{1}{2}}
$$

In the above expression, $w$ and $\alpha$ are respectively the electron impact parameter and the ion broadening parameter tabulated by Griem [29], $R$ (the Debye shielding parameter) is defined as the ratio of the mean inter-ion distance to the Debye radius. This expression is sufficiently accurate as long as the following conditions are fulfilled:

$$
R \leqslant 0.8, \quad 0.05 \leqslant \alpha \leqslant 0.5 .
$$

It is important to note that this measurement weakly depends on the electron temperature and is completely independent of the thermodynamic state of the plasma. 


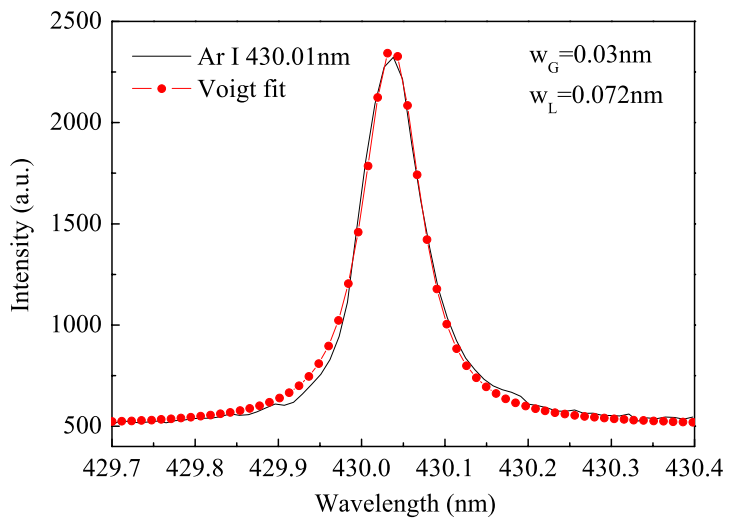

Figure 11. Typical Voigt fit of the recorded Ar I $430.01 \mathrm{~nm}$ line (at $10 \mathrm{~mm}$ downstream from the torch exit).

Goldbach et al [30] represented the following formula to determine the electron density. The linear dependence of the Stark width $\Delta \lambda_{\mathrm{S}}$ of two Ar I lines $(415.86,430.01 \mathrm{~nm})$ upon the electron density $n_{\mathrm{e}}\left(\mathrm{m}^{-3}\right)$ is obtained by fitting of all the experimental data

$$
\Delta \lambda_{\mathrm{S}}=C n_{\mathrm{e}}
$$

where $C$ is a broadening coefficient given in the literature [30]. For the Ar I $415.86 \mathrm{~nm}$ line, $C=2.3 \times 10^{-24} \mathrm{~nm} \mathrm{~m}^{3}$; for Ar I $430.01 \mathrm{~nm}, C=2.27 \times 10^{-24} \mathrm{~nm} \mathrm{~m}^{3}$.

In addition, a curve-fitted empirical formula for calculating the electron density $n_{\mathrm{e}}\left(\mathrm{m}^{-3}\right)$ by using the Stark width $\Delta \lambda_{\mathrm{S}}$ of the $\operatorname{Ar} \mathrm{I}(430.01 \mathrm{~nm})$ line is presented by Deron [24]:

$$
\ln n_{\mathrm{e}}=58.241+0.992 \ln \Delta \lambda_{\mathrm{S}}-0.414 \ln T_{\mathrm{e}} .
$$

Figure 11 displays a typical recorded Ar I $415.86 \mathrm{~nm}$ line profile with a calculated Voigt one. The best fit between these profiles leads to the value of the Stark width, which allows us to calculate the electron density using three different formulae. The electron densities calculated from the argon atom emission are very consistent when using different emission lines and calculation formulae.

The results show that the electron density also exhibits a falling evolution along the jet axis, decreasing from $7.6 \times 10^{22} \mathrm{~m}^{-3}$ inside the arc chamber to $3.1 \times 10^{22} \mathrm{~m}^{-3}$ at $10 \mathrm{~mm}$ downstream of the torch exit. The uncertainty of this method can be estimated as better than $10 \%$.

3.4.3. Local thermodynamic equilibrium. Several theoretical and experimental studies have been performed to investigate the validity criteria of LTE existing in the plasma sources [29, 31-37]. Many of these criteria are based on the determination of a minimum electron density.

In an optically thin plasma, if the collision processes are more predominant than the radiation decay and the selfabsorption of the resonance lines can be neglected, the threshold value of the electron density for the LTE state is proposed by Griem [29,31]:

$$
n_{\mathrm{e}}\left(m^{-3}\right) \geqslant 9.2 \times 10^{23}\left(\frac{k_{\mathrm{B}} T}{E_{\mathrm{H}}}\right)^{\frac{1}{2}}\left(\frac{E_{2}-E_{1}}{E_{\mathrm{H}}}\right)^{3},
$$

where $E_{\mathrm{H}}$ is the ionization energy of the hydrogen atom, $E_{2}-E_{1}$ is the energy gap between the first excited level and the ground level.

For the electron temperature of a plasma arc around $10000 \mathrm{~K}$, according to equation (10), the LTE could exist if the electron density fulfils: $n_{\mathrm{e}} \geqslant 1.1 \times 10^{23} \mathrm{~m}^{-3}$. This threshold obtained by Griem's criterion is very high. Numerous studies indicate that Griem overestimated the relevant electron density threshold. This is connected primarily with the assumption that radiation of resonance transitions freely leaves a given volume. Owing to the considerable value of the absorption coefficient for the resonance lines, this assumption, however, is never fulfilled. The theory of the influence of the radiation re-absorption on the deviation from the LTE, developed for hydrogen and helium by Biberman et al [32] and Drawin [33], suggested that the LTE could be expected to prevail in a plasma at a much lower electron density (by an order of magnitude) than theory predicts. Kolesnikov [35] have determined deviations from the LTE in an argon plasma arc by measuring various 'temperatures': population temperature $\left(T_{\mathrm{p}}\right)$, distribution temperature $\left(T_{\mathrm{d}}\right)$, ionization temperature $\left(T_{\mathrm{i}}\right)$, gas temperature $\left(T_{\mathrm{g}}\right)$ and electron temperature $\left(T_{\mathrm{e}}\right)$. The results show that the LTE exists for electron densities greater than $5 \times 10^{21} \mathrm{~m}^{-3}$. Other works carried out by Novak [36], Richter [37] and Bourasseau et al [38] also lead to the conclusion that the LTE can be assumed at electron densities of a few times $10^{22} \mathrm{~m}^{-3}$.

Based on further considerations and detailed computer calculations, Griem represented a new criterion in which the self-absorption of the resonance radiation is taken into account $[29,31]$ :

$$
n_{\mathrm{e}} \geqslant 1 \times 10^{23}\left(\frac{k_{\mathrm{B}} T}{E_{\mathrm{H}}}\right)^{\frac{1}{2}}\left(\frac{E_{2}-E_{1}}{E_{\mathrm{H}}}\right)^{3}\left[\mathrm{~m}^{-3}\right] .
$$

Assuming an electron temperature of $10000 \mathrm{~K}$ in an argon plasma jet, the minimum value of $n_{\mathrm{e}}$ obtained by equation (11) for the LTE is $1.4 \times 10^{22} \mathrm{~m}^{-3}$. Consequently, in our standard conditions, the electron densities inside the arc chamber and at the torch exit satisfy the criterion. It suggests that the composition of the plasma may be expected to be close to that deduced from an LTE calculation.

A simple LTE model involving Dalton's law, the electroneutrality condition and mass action laws (Saha-Eggert equation) is performed to determine the plasma composition at atmospheric pressure. The following four species are taken into account: $\mathrm{Ar}, \mathrm{Ar}^{+}, \mathrm{Ar}^{++}$and $\mathrm{e}^{-}$. The results of this calculation are compared with the measured values of the electron density.

The composition of an argon plasma arc in the LTE state is determined from the following set of equations.

Dalton's law $\quad \frac{P}{k_{\mathrm{B}} T}=n_{\mathrm{e}}+n_{\mathrm{Ar}}+n_{\mathrm{Ar}^{+}}+n_{\mathrm{Ar}^{++}}$,

Electroneutrality $\quad n_{\mathrm{e}}=n_{\mathrm{Ar}^{+}}+2 n_{\mathrm{Ar}^{++}}$,

Saha-Eggert equation $\frac{n_{\mathrm{e}} n_{\mathrm{Ar}^{+}}}{n_{\mathrm{Ar}}}$

$$
=\frac{g_{\mathrm{e}} g_{\mathrm{Ar}^{+}}}{g_{\mathrm{Ar}}}\left[\frac{2 \pi m_{\mathrm{e}} k_{\mathrm{B}} T}{h^{2}}\right]^{3 / 2} \exp \left(-\frac{E_{\mathrm{Ar}^{+}}}{k_{\mathrm{B}} T}\right),
$$




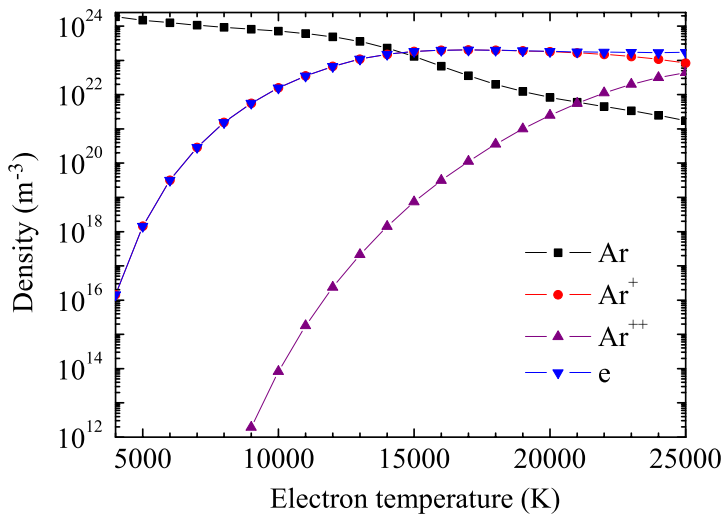

Figure 12. Composition of the argon plasma arc in the LTE state at atmospheric pressure.

$$
\begin{aligned}
& \text { Saha-Eggert equation } \frac{n_{\mathrm{e}} n_{\mathrm{Ar}^{++}}}{n_{\mathrm{Ar}^{+}}} \\
& =\frac{g_{\mathrm{e}} g_{\mathrm{Ar}^{++}}}{g_{\mathrm{Ar}^{+}}}\left[\frac{2 \pi m_{\mathrm{e}} k_{\mathrm{B}} T}{h^{2}}\right]^{3 / 2} \exp \left(-\frac{E_{\mathrm{Ar}^{++}}}{k_{\mathrm{B}} T}\right),
\end{aligned}
$$

where $E_{\mathrm{Ar}^{+}}=15.755 \mathrm{eV}$ and $E_{\mathrm{Ar}^{++}}=27.76 \mathrm{eV}$ are the ionization energies of $\mathrm{Ar}^{+}$and $\mathrm{Ar}^{++}$, respectively. The lowering $\Delta E_{\text {ion }}$ of these energies can be calculated from the model of Coulomb interaction developed by Griem [39]:

$$
\Delta E_{\mathrm{ion}}=\frac{(z+1) e^{2}}{4 \pi \varepsilon_{0} \lambda_{\mathrm{D}}},
$$

where $z$ is the charged number and $\lambda_{D}$ is the Debye radius.

In our conditions, $\Delta E_{\mathrm{ion}}$ is negligible. For instance, the Ar ionization potential lowering is only $0.06 \mathrm{eV}$ in contrast to $E_{\mathrm{Ar}^{+}}=15.755 \mathrm{eV}$ for $n_{\mathrm{e}}=1 \times 10^{23} \mathrm{~m}^{-3}$ and $T_{\mathrm{e}}=10000 \mathrm{~K}$.

Figure 12 shows the composition of atmospheric argon plasma arc in the LTE state. As can be seen from figure 12, in an LTE argon plasma at $12300 \mathrm{~K}$, the major species are clearly $\mathrm{Ar}, \mathrm{Ar}^{+}$and free electrons, and the number density of these species:

$$
\begin{gathered}
n_{\mathrm{e}}=7.9 \times 10^{22} \mathrm{~m}^{-3}, \quad n_{\mathrm{Ar}^{+}}=7.9 \times 10^{22} \mathrm{~m}^{-3}, \\
n_{\mathrm{Ar}}=4.6 \times 10^{23} \mathrm{~m}^{-3} .
\end{gathered}
$$

The calculated electron density is quite compatible with the measured one at the divergent part of the first anode, confirming well that the plasma is actually close to equilibrium in this zone.

When the temperature is fixed at the value $(10500 \mathrm{~K})$ which is measured at the exit of the plasma torch, the same computation from the LTE model leads to the following composition:

$$
\begin{gathered}
n_{\mathrm{e}}=2.4 \times 10^{22} \mathrm{~m}^{-3}, \quad n_{\mathrm{Ar}^{+}}=2.4 \times 10^{22} \mathrm{~m}^{-3}, \\
n_{\mathrm{Ar}}=6.6 \times 10^{23} \mathrm{~m}^{-3} .
\end{gathered}
$$

The theoretical electron density is not so far from the one derived from the Stark broadening of ArI lines: $n_{\mathrm{e}}=$ $3.1 \times 10^{22} \mathrm{~m}^{-3}$. Therefore, the double arc argon plasma is still quite close to the LTE state at the torch exit.

In summary, the electron densities deduced from the LTE model are quite compatible with those obtained using the Stark broadening of ArI lines. As expected, the atmospheric dc argon plasma jet at the double arc mode is close to the LTE state under our experimental conditions. The local gradients of the plasma properties (electron temperature and density) along the jet axis are smoothly varied, which gives diffused particles enough time to get to the equilibrium conditions. It also indicates that the ignition of the second arc prevents the strong collapse of the ionization which is usually observed in the classical plasma torch.

\section{Conclusion}

This work is devoted to the study of the characterization of an atmospheric argon plasma jet generated by a dc plasma torch with a double anode structure. Such an original configuration has been proved to be effective in enhancing the aerodynamic stability and luminous intensity of the plasma as well as the jet length. In addition, this torch with special design also provides the possibility of maintaining a much higher arc voltage and input power at relatively low current intensity.

The current-voltage characteristics of atmospheric double arc argon plasma show an increasing tendency. From the fluctuations of electrical signals coupled with their statistical analysis, the takeover mode is identified as the fluctuation characteristic of the argon plasma jet. Whatever the operating conditions, the FFT and correlation results of electrical signals exhibit the only prominent peak of $150 \mathrm{~Hz}$, whereas no high frequency components $(1-15 \mathrm{kHz})$ are found. It indicates that the arc fluctuations of the argon plasma jet are mainly attributed to the undulation of the tri-phase rectified power supply. In addition, both arcs behave as the one and only system, and each arc root attachment is evenly diffused rather than located at a fixed position on the anode channel. Such an arc attachment mode is favorable for reducing the anode wear and offering a long working time for the electrodes.

The spectroscopic diagnostic technique is employed to measure the emission spectra of the argon plasma jet inside and outside the arc chamber. In our experiments, the excitation temperatures of the plasma arc are determined by using the Boltzmann plot method and are expected to be close to the electronic ones, while the electron number densities are derived from the Stark broadening of Ar I lines. The axial evolution shows that the measured electron temperature and density of the argon plasma drop along the jet axis from $12300 \mathrm{~K}$ and $7.6 \times 10^{22} \mathrm{~m}^{-3}$ in the divergent part of the first anode nozzle to $10500 \mathrm{~K}$ and $3.1 \times 10^{22} \mathrm{~m}^{-3}$ at the torch exit. Thus, the decrease of the electron parameters (temperature and density) is much smoother than the one of standard torch plasmas. Therefore, the plasma torch with a second anode configuration effectively reduces the arc instabilities and prevents strong decay of the plasma excitation between these lines of sight.

Furthermore, a simple LTE model involving Dalton's law, the electroneutrality condition and mass action laws is developed to calculate the plasma composition at atmospheric pressure. The results show that the measured electron densities are quite compatible with those obtained from the LTE model, which indicates that the double arc argon plasma is very close to the LTE state under our experimental conditions.

\section{Acknowledgments}

This work was financially supported by the Sino-France Program for Advanced Research (Grant No PRA E01-06) 
and the Major Program of International Cooperation from the Science and Technology Department of Zhejiang Province, China (Grant No 2005C14014).

\section{References}

[1] Fauchais P and Vardelle A 1997 IEEE Trans. Plasma Sci. 251258

[2] Pfender E 1999 Plasma Chem. Plasma Process. 191

[3] Coudert J F, Planche M P and Fauchais P 1996 Plasma Chem. Plasma Process. 16 S211

[4] Singh N, Razafinimanana M and Hlina J 2000 J. Phys. D: Appl. Phys. 33270

[5] Dorier J L, Gindrat M, Hollenstein C, Salito A, Loch M and Barbezat G 2001 IEEE Trans. Plasma Sci. 29494

[6] Duan Z and Heberlein J 2002 J. Thermal Spray Technol. 1144

[7] Benocci R, Florio R, Galassi A, Paolicchio M, Piselli M, Sala C, Sciascia M and Sindoni E 1999 Eur. Phys. J. D 6269

[8] Zhao W H, Tian K, Tang H Z, Liu D and Zhang G Z 2002 J. Phys. D: Appl. Phys. 352815

[9] Joshi N K, Sahasrabudhe S N, Sreekumar K P and Venkatramani N 2003 Eur. Phys. J. D 26215

[10] Yugeswaran S and Selvarajan V 2006 Vacuum 81347

[11] Benmansour M, Nikravech M, Morvan D, Amouroux J and Chapelle J 2004 J. Phys. D: Appl. Phys. 372966

[12] Konjevic N 2001 Plasma Sources Sci. Technol. 10356

[13] Fantz U 2006 Plasma Sources Sci. Technol. 15 S137

[14] Belevtsev A A, Chinnov V F and Isakaev E Kh 2006 Plasma Sources Sci. Technol. 15450

[15] Rajabian M, Gravelle DV and Vacquie S 2004 Plasma Chem. Plasma Process. 24285

[16] Robin L, Chéron B G and Vervish P 1993 Phys. Fluids B 5610

[17] Robin L, Vervish P and Chéron B G 1994 Phys. Plasmas 1444
[18] Bultel A, Vervish P and Chéron B G 1995 Plasma Sources Sci. Technol. 4597

[19] Delair L, Tu X, Bultel A and Chéron B G 2005 High Temp. Mater. Process. 9583

[20] Osaki K, Fukumasa O and Kobayashi A 2000 Vacuum 5947

[21] Pan W X, Zhao W H, Ma W and Wu C K 2002 Plasma Chem. Plasma Process. 22271

[22] Delair L 2004 Ph.D thesis, Universitéde Rouen, Rouen, France

[23] Hrabovsky M, Kopecky V, Sember V, Kavka T, Chumak O and Konrad M 2006 IEEE Trans. Plasma Sci. 341566

[24] Deron C 2003 PhD thesis, Ecole Central de Paris

[25] Kaminska A and Dudeck M 1998 High Temp. Mater. Process. 2117

[26] Dorier J L, Hollenstein C, Salito A, Loch M and Barbezat G 1997 14th Int. Symp. Plasma Chemistry (Prague, Czech Republic) p 331

[27] De Izarra C, Vallee O and Pauvit R 1999 High Temp. Mater. Process. 3213

[28] NIST Atomic Spectra Database Data, http://physics.nist.gov/

[29] Griem H R 1964 Plasma Spectroscopy (New York: McGraw-Hill)

[30] Goldbach C, Nollez G and Plomdeur P 1977 J. Phys. B: At. Mol. Opt. Phys. 101181

[31] Griem H R 1963 Phys. Rev. 1311170

[32] Biberman L M, Vorobev V S and Lagarkov A N 1965 opt. Spectroscopy (USSR) 19326

[33] Drawin H W 1969 Z. Naturforsch. 24a 1492

[34] Drawin H W 1970 High Press.-High Temp. 2359

[35] Kolesnikov V N 1966 Phys. Opt. 3053

[36] Novak J P 1976 J. Quant. Spectrosc. Radiat. Transfer 17247

[37] Richter J 1965 Z. Astrophysik 6157

[38] Bourasseau D, Cabannes F and Chapelle J 1970 Astron. Astrophs. 9339

[39] Griem H R 1974 Spectral Line Broadening by Plasma (New York: Academic) 